\title{
Long-Term Potentiation Is Impaired in Middle-Aged Rats: Regional Specificity and Reversal by Adenosine Receptor Antagonists
}

\author{
Christopher S. Rex, ${ }^{1}$ Enikö A. Kramár, ${ }^{2}$ Laura L. Colgin, ${ }^{2}$ Bin Lin, ${ }^{2}$ Christine M. Gall, ${ }^{1,3}$ and Gary Lynch ${ }^{2}$ \\ ${ }^{1}$ Department of Neurobiology and Behavior, University of California, Irvine, California 92697-4550, 2Department of Psychiatry and Human Behavior, \\ University of California, Irvine, California 92612-1695, and ${ }^{3}$ Department of Anatomy and Neurobiology, University of California, Irvine, California 92697- \\ 1275
}

Memory loss in humans begins early in adult life and progresses thereafter. It is not known whether these losses reflect the failure of cellular processes that encode memory or disturbances in events that retrieve it. Here, we report that impairments in hippocampal long-term potentiation (LTP), a form of synaptic plasticity associated with memory, are present by middle age in rats but only in select portions of pyramidal cell dendritic trees. Specifically, LTP induced with theta-burst stimulation in basal dendrites of hippocampal field CA1 decayed rapidly in slices prepared from 7- to 10-month-old rats but not in slices from young adults. There were no evident age-related differences in LTP in the apical dendrites. Both the adenosine $A_{1}$ receptor antagonist 8-cyclopentyl-1,3-dipropylxanthine and a positive AMPA receptor modulator (ampakine) offset age-related LTP deficits. Adenosine produced greater depression of synaptic responses in middle-aged versus young adult slices and in basal versus apical dendrites. These results were not associated with variations in $A_{1}$ receptor densities and may instead reflect regional and age-related differences in adenosine clearance. Pertinent to this, brief applications of $A_{1}$ receptor antagonists immediately after theta stimulation fully restored LTP in middle-aged rats. We hypothesize that the build-up of extracellular adenosine during theta activity persists into the postinduction period in the basal dendrites of middle-aged slices and thereby activates the $A_{1}$ receptor-dependent LTP reversal effect. Regardless of the underlying mechanism, the present results provide a candidate explanation for memory losses during normal aging and indicate that, with regard to plasticity, different segments of pyramidal neurons age at different rates.

Key words: LTP; basal dendrites; hippocampus; DPCPX; aging; A1 adenosine receptor; theta-burst stimulation; reversal

\section{Introduction}

Memory losses in humans are not restricted to old age but instead begin to emerge much earlier in life. One recent study found progressively greater impairments across subjects from 30 to 90 years of age (Davis et al., 2003), whereas another reported a steady decrease across multiple measures of memory in healthy adults from age 20 forward (Park et al., 2003). Declines in memory from young adulthood to middle age have also been described previously for rats (Deupree et al., 1993; Granger et al., 1996; Oler and Markus, 1998; Ward et al., 1999; Meneses et al., 2004) and monkeys (Herndon et al., 1997; Sloane et al., 1997), suggesting that this effect could be a general feature of mammalian brain. These observations raise the question of whether long-term potentiation (LTP), a form of synaptic plasticity widely regarded as a substrate of memory encoding, also begins to deteriorate relatively early in adult life.

\footnotetext{
Received Nov. 23, 2004; revised April 20, 2005; accepted May 14, 2005.

This work was supported by National Institute on Aging Grant AG00358 and National Institute of Mental Health (NIMH) Grant MH61007. C.S.R. and L.L.C. were supported by NIMH Training Grants 5-T32-MH14599-27 and T32 AG00096-21, respectively.

Correspondence should be addressed to Christine M. Gall, 3226 Gillespie Neuroscience Research Facility, University of California, Irvine, CA 92697. E-mail: cmgall@uci.edu.

D01:10.1523/JNEUROSCI.0880-05.2005

Copyright $\odot 2005$ Society for Neuroscience $\quad$ 0270-6474/05/255956-11\$15.00/0
}

Several studies describe deficits in LTP in aged ( $\sim 2$ years) rodents (Barnes, 1979; Barnes and McNaughton, 1985; Deupree et al., 1993; Shankar et al., 1998). Other work indicates that this does not hold across all conditions (Diana et al., 1994; Norris et al., 1996) and that the presence and magnitude of deficits depends on the stimulation patterns used to induce potentiation (Moore et al., 1993; Lanahan et al., 1997; Watabe and O'Dell, 2003) and whether the animals were deficient in learning (Tombaugh et al., 2002). There appear to be no reports of impaired LTP in middle-aged rodents. This could indicate that such deficits are not present and that middle-age memory impairments are attributable to factors other than a loss of synaptic plasticity. However, the search for age-related changes in LTP has focused on the dentate gyrus and CA1 stratum (str.) radiatum; it is not known whether results from these areas can be generalized to other regions of forebrain or even to other subfields of hippocampus.

Possibly relevant to this, there are clear differences between hippocampal subregions with regard to properties of LTP. Potentiation is more easily induced and greater in magnitude in the basal dendrites (stratum oriens) compared with the apical dendrites (stratum radiatum) of field CA1 (Capocchi et al., 1992; Kaibara and Leung, 1993; Arai et al., 1994; Roth and Leung, 1995; Leung and Shen, 1999; Kramár and Lynch, 2003). These withincell variations appear to be caused by reduced feedforward IPSPs 
during theta-burst responses and smaller burst afterhypolarizations in the stratum oriens (Arai et al., 1994). The latter effect has been traced to apical/basal differences in potassium channels (Sah and Bekkers, 1996). More recent work has uncovered differences in LTP stabilization between the two dendritic domains: apical dendritic potentiation becomes resistant to reversal within 15-30 min after induction (Barrionuevo et al., 1980; Arai et al., 1990a,b), whereas basal dendritic LTP remains vulnerable for $>1$ h (Kramár and Lynch, 2003).

The present studies tested whether apical/basal differences extend to the effects of aging on LTP. We report here that potentiation is indeed significantly impaired in the stratum oriens, but not stratum radiatum, in middle-aged rats and that this deficit is associated with age effects on adenosine processing.

\section{Materials and Methods}

Hippocampal slices and subfield dissections. Young (1-2 months of age) or middle-aged (7-10 months of age) adult male Sprague Dawley rats (Charles River Laboratories, Wilmington, MA) were anesthetized with halothane and decapitated following protocols approved by the University of California Institutional Animal Care and Use Committee. Brains were quickly removed and placed in ice-cold oxygenated dissection medium containing the following (in $\mathrm{mM}$ ): $124 \mathrm{NaCl}, 3 \mathrm{KCl}, 1.25 \mathrm{KH}_{2} \mathrm{PO}_{4}$, $5 \mathrm{MgSO}_{4}, 26 \mathrm{NaHCO}_{3}, 3.4 \mathrm{CaCl}_{2}$, and 10 dextrose. Unless otherwise noted, chemicals were purchased from Sigma (St. Louis, MO). Transverse hippocampal slices ( $350 \mu \mathrm{m}$ thick) were cut through the middle third of the septotemporal arc of the hippocampus using a vibrating tissue slicer (VT1000; Leica, Nussloch, Germany). Slices used for electrophysiological recording were placed in an interface recording chamber maintained at $30 \pm 1.0^{\circ} \mathrm{C}$ and humidified with $95 \% \mathrm{O}_{2} / 5 \% \mathrm{CO}_{2}$. Oxygenated artificial CSF (aCSF) containing (in mM) $124 \mathrm{NaCl}, 3 \mathrm{KCl}, 1.25$ $\mathrm{KH}_{2} \mathrm{PO}_{4}, 2.5 \mathrm{MgSO}_{4}, 26 \mathrm{NaHCO}_{3}, 3.4 \mathrm{CaCl}_{2}$, and 10 dextrose was infused at a rate of $60-70 \mathrm{ml} / \mathrm{h}$. Slices were allowed at least $1 \mathrm{~h}$ to equilibrate to the chamber environment before testing. When performing subfield dissections, five slices were kept at $32^{\circ} \mathrm{C}$ in dissection medium $(<15 \mathrm{~min})$ before being placed on an agarose block under a dissecting microscope, where the CA1 region was isolated using a scalpel. The stratum oriens and str. radiatum were then isolated, and laminar samples from each rat were pooled and stored at $-80^{\circ} \mathrm{C}$ for later Western blot analysis.

Electrophysiology. To assess field EPSPs (fEPSPs) in the apical and basal dendritic fields of the CA1 pyramidal cells (i.e., the strata radiatum and oriens, respectively), orthodromic stimulation (twisted nichrome wires, $65 \mu \mathrm{m}$ ) was delivered to the Schaffer collateral/commissural afferents in CAla or CA1c (str. radiatum or oriens), and responses were recorded within the same lamellas of CA1b using a single glass pipette (electrode resistance, $5 \mathrm{M} \Omega$ ) filled with $2 \mathrm{M} \mathrm{NaCl}$. Recording electrodes for the str. oriens were situated at the boundary between the str. oriens and the alveus, whereas those in the str. radiatum were located at an equivalent distance from the str. pyramidale. Input-output curves were conducted before LTP induction by raising stimulus duration while maintaining a constant intensity of $35 \mu \mathrm{A}$. Baseline measures (20-30 min) were established using single-pulse stimulation delivered at $30 \mathrm{~s}$ intervals with current intensity adjusted to $50 \%$ of the maximum, spike-free fEPSP for the str. radiatum and $20-40 \%$ for the str. oriens. Synaptic potentiation was induced using theta-burst stimulation (TBS) (Larson and Lynch, 1986; Larson et al., 1986) consisting of 10 bursts of four $100 \mathrm{~Hz}$ pulses, with each burst separated by $200 \mathrm{~ms}$. The duration of stimulation pulses to the str. oriens, but not the str. radiatum, was increased by $20-40 \%$ during the TBS period. fEPSP slope (measured at $20-80 \%$ fall of the slope) and amplitude were measured from digitized EPSPs (NacGather 2.0; Theta Burst, Irvine, CA) and normalized to the $10 \mathrm{~min}$ baseline or drug infusion period directly preceding manipulation (i.e., TBS, drug infusion). All measures are reported and illustrated as mean \pm SEM. Responses to individual theta bursts were recorded and analyzed as the burst area below baseline level. The sizes of responses (composite area) to bursts 2-10 are presented as a percentage change from the area of the initial burst. The area of the initial burst response is presented as a percentage change from the averaged areas of fEPSPs recorded during the $5 \mathrm{~min}$ period preceding TBS.

Drug application. Unless otherwise indicated, drugs were introduced to the bath via a second perfusion line of silicon tubing. Perfusion flow was induced by gravity and controlled by an intravenous regulator. Adenosine (EMD Biosciences, San Diego, CA), the adenosine $A_{1}$ receptor $\left(A_{1} R\right)$ antagonist 8-cyclopentyl-1,3-dipropylxanthine (DPCPX; Tocris Cookson, Ellisville, MO), or the positive AMPA receptor modulator CX614 (Cortex Pharmaceuticals, Irvine, CA) was delivered to the recording chamber after a stable aCSF baseline was obtained (or, in cases in which multiple drugs were applied, after a stable drug infusion baseline was obtained). Drug stocks were prepared with $100 \%$ dimethylsulfoxide (DMSO) and stored at $4^{\circ} \mathrm{C}$. Immediately preceding each experiment, drugs were diluted to their final working concentrations in aCSF containing $\leq 0.005 \%$ DMSO. The DPCPX experiments shown in Figure 6 used a drug-filled glass micropipette (tip diameter, $\sim 25 \mu \mathrm{m}$ ) lowered into the bath solution upstream from the recording slice. The micropipette was attached to a $10 \mathrm{ml}$ syringe, and flow $(0.2 \mathrm{ml} / \mathrm{min})$ was controlled by a motorized injection pump (Sage Instruments, Freedom, CA). The concentration of DPCPX in the micropipette was $2.5 \mu \mathrm{M}$. This concentration is approximately five times higher than the final desired concentration, to compensate for dilution of the drug solution after entering the slice chamber.

Immunohistochemistry. Male Sprague Dawley rats were anesthetized with sodium pentobarbital and intracardially perfused with $4 \%$ paraformaldehyde in $0.1 \mathrm{M}$ phosphate buffer (PB), $\mathrm{pH} 7.2$. The brain was removed, postfixed at $4^{\circ} \mathrm{C}$ for $3-5 \mathrm{~h}$, cryoprotected in $20 \%$ sucrose in $\mathrm{PB}$ for $48 \mathrm{~h}\left(4^{\circ} \mathrm{C}\right)$, and sectioned at $30 \mu \mathrm{m}$ on a freezing microtome; sections were cut on the same plane and through the same region as those for slices used for electrophysiological analyses. The tissue was pretreated with $2 \mu \mathrm{g} / \mathrm{ml}$ proteinase $\mathrm{K}\left(37^{\circ} \mathrm{C} ; 10 \mathrm{~min}\right)$ in $10 \mathrm{~mm}$ Tris- $\mathrm{HCl}$ containing $1 \mathrm{~mm}$ EDTA, rinsed through several changes of $0.1 \mathrm{~m}$ Tris-buffered saline (tbs), and processed for immunohistochemical localization of $\mathrm{A}_{1}$ adenosine receptor using affinity-purified polyclonal antisera (\#A-268; Sigma), the Vectastain Elite ABC kit from Vector Laboratories (Burlingame, $\mathrm{CA}$ ), and 3,3'-diaminobenzidine (DAB) as chromagen. The primary antisera were used at 1:1000 dilution in tbs (containing $1.5 \%$ bovine serum albumin and $0.1 \%$ Triton $\mathrm{X}-100$ ) and incubated overnight at room temperature. The $\mathrm{DAB}$ reaction mixture included $0.035 \% \mathrm{CoCl}_{2}$, $0.02 \%$ nickel ammonium sulfate, and $0.6 \% \mathrm{H}_{2} \mathrm{O}_{2}$ in $\mathrm{PB}$. After the DAB reaction, the tissue was mounted onto Superfrost microscope slides (Fisher Scientific, Pittsburgh, PA) and coverslipped with Permount (Fisher Scientific).

Immunoblotting. Laminar tissue samples were homogenized in radioimmunoprecipitation assay buffer containing $10 \mathrm{~mm}$ Tris, $\mathrm{pH}$ 7.2, 158 mм NaCl, 1 mм EDTA, $0.1 \%$ SDS, $1 \%$ sodium deoxycholate, $1 \%$ Triton X-100, $1 \times$ Complete Protease Inhibitor Cocktail (Roche Diagnostics, Mannheim, Germany), and Phosphatase Inhibitor Cocktails 1 and 2 (P2850 and 5726; Sigma). Sample volumes were adjusted to normalize protein content as determined by the Bio-Rad (Hercules, CA) protein assay and separated by $4-12 \%$ SDS-PAGE. For Western blot analysis, proteins were transferred to polyvinylidene difluoride membrane (Amersham Biosciences, Piscataway, NJ) and processed with antisera to adenosine $\mathrm{A}_{1}$ receptor (\#A-268, as above; 1:1500) and to the PDZ [postsynaptic density-95 (PSD-95)/Discs large/zona occludens-1] domain of PSD-95 (clone K28/86.2; 1:1500; Upstate Biotechnology, Lake Placid, $\mathrm{NY}$ ) in tbs containing 5\% bovine serum albumin using the ECL Plus system (Amersham Biosciences) as described previously (Kramár et al., 2003). Immunoreactivity was quantified by densitometric analysis of film using the Analytical Imaging Station of Imaging Research (St. Catharines, Ontario, Canada).

\section{Results \\ Region-specific impairments in LTP in the hippocampus of middle-aged rats}

Responses elicited by single-pulse stimulation of the Schaffer collateral/commissural projections to the apical and basal dendrites of field CA1 in hippocampal slices from young (1-2 months of 
age) and middle-aged (7-10 months of age) rats revealed no evident age differences in the waveforms of the field EPSPs and mean values for slopes, amplitudes, and half-widths. An examination of input-output curves, shown in Figure 1, $A$ and $B$, found that the mean amplitudes of field EPSPs in young adult versus middleaged slices were comparable across a range of stimulation pulse durations in both apical (two-way repeated-measures ANOVA; $p>0.6)$ and basal $(p>0.6)$ dendrites. Paired-pulse facilitation (Fig. 1C) was not detectably different in either str. oriens (two-tailed $t$ test; $p>0.2$ at 10,50 , and 100 ms pulse intervals) or str. radiatum $(p>$ 0.3 at all pulse intervals) between the two age groups. Baseline responses recorded with whole-cell clamp (Kramár et al., 2004) also appeared comparable with regard to rise times, decay time constants, and amplitudes of the EPSCs (data not shown). Whole-cell recording also indicated that the afterhyperpolarizations (AHPs) that follow a theta burst, and have been linked to the magnitude of LTP (Arai and Lynch, 1992), were well developed in middle-aged slices and comparable with those found in young adults. Figure $1 D$ shows typical clamp records from cases in which a single theta burst was delivered to the Schaffer-commissural projections in the CA1 basal dendrites in slices from 9-month-old rats to elicit an AHP. Analysis of group data (Fig. $1 E$ ) did not uncover evidence for reliable age-related differences in AHP amplitudes; differences in mean values for young adult versus middle-aged cases did not achieve statistical significance (two-tailed $t$ test; $p>0.2$ ), and median scores for the two groups were almost identical.

Although all of the physiological measures described above were found to be similar in the young adult and middle-aged groups, striking differences were observed in the stability of LTP in the basal dendrites. TBS caused an immediate and equivalent increase in fEPSP slope in the basal dendritic field of young adult ( 1 min after TBS, $153 \pm 13 \%$ increase over baseline) and middleaged ( 1 min after TBS, $165 \pm 13 \%$ ) slices (Fig. 2 A). Potentiation in the str. oriens of slices from young adults decayed steadily for $\sim 15$ min and stabilized at a level that was $81 \pm 11 \%$ above baseline; this increment in response size was maintained for the remainder of the recording period. Figure $2 B$ summarizes data from four experiments in which responses were followed for 60 min after TBS; as shown, potentiation for this subgroup remained $>75 \%$ above baseline. LTP in the middle-aged slices, although equivalent through the first $10 \mathrm{~min}$ after induction, decayed steadily after that point, thus, by $30 \mathrm{~min}$, it was $44 \pm 10 \%$ above baseline (Fig. $2 \mathrm{~A}$ ), a value that was substantially less than that in the young adult group $(80 \pm 11 \%$ above baseline; $p<$ 0.05 ; two-tailed $t$ test). The disparity between age groups increased at later time points (Fig. $2 B$ ), because potentiation persisted in young adults but continued to decline in the older animals $(60 \mathrm{~min}$ after TBS, $+76 \pm 13$ vs $+23 \pm 18 \% ; p<0.01$;
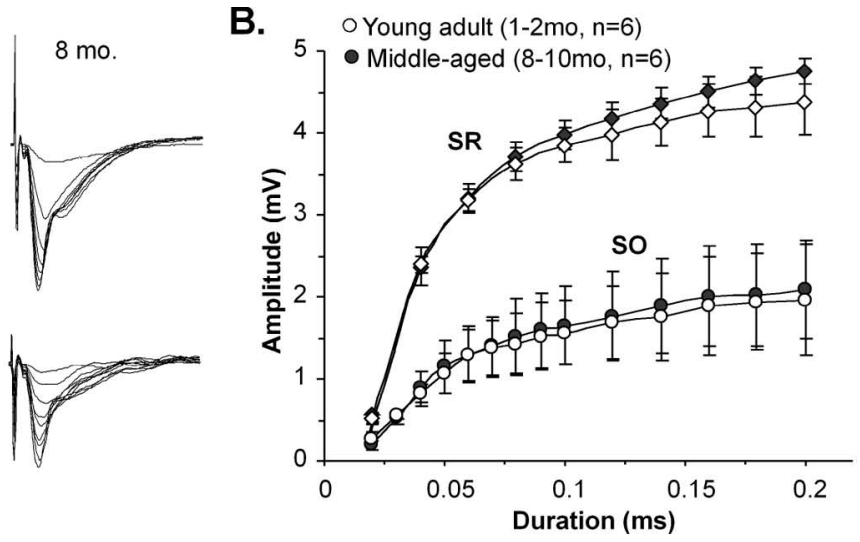

D.

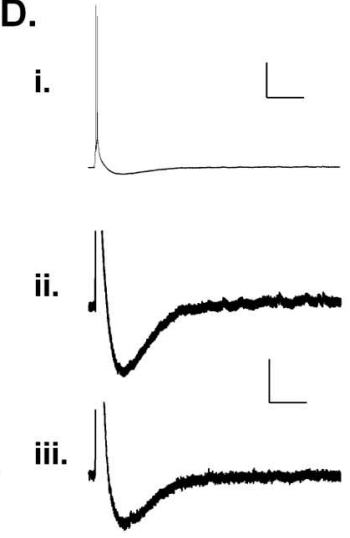

E. Middle age $(n=10)$
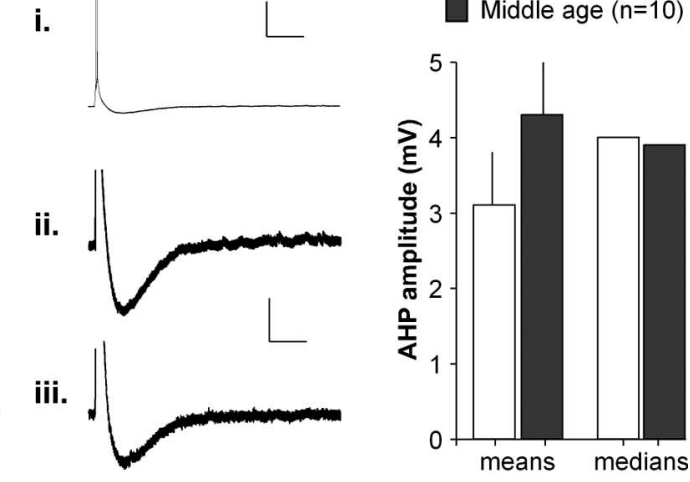
$\square$ Young adult $(n=7)$

.

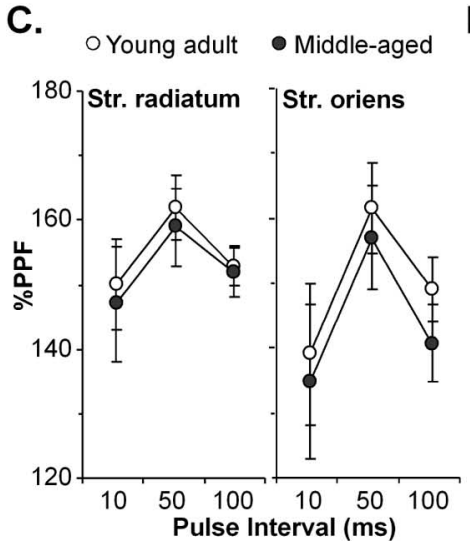

Figure 1. Young adult and middle-aged Schaffer-commissural responses are comparable by several measures. $\boldsymbol{A}$, Traces from representative input- output experiments comparing fEPSP responses within the stratum radiatum $(S R)$ and stratum oriens (SO) 列 50 , and 100 ms pulse intervals showed no differences between slices from young adult and middle-aged rats. D, Representative $2.5 \mathrm{mV}, 0.5 \mathrm{~s}$. $\boldsymbol{E}$, Comparison of mean (left) AHP amplitudes, measured from EPSC $s$ of young adult and middle-aged slices, reveals no significant age-related differences ( $p>0.2$; two-tailed $t$ test). Median scores (right) are nearly identical.

two-way repeated-measures ANOVA for minutes 50-60). Even when a more robust induction paradigm was used (i.e., stimulus duration doubled during the TBS train), middle-aged slices still exhibited LTP deficits at 30 and 60 min after TBS that were equivalent to those reported above (data not shown).

Analysis of responses to TBS trains has proven to be an effective tool for uncovering factors that could be responsible for modifications in LTP (Kramár et al., 2004) and was used for this purpose in the present experiments. There were no obvious age differences in the shape of the burst responses in the str. oriens (Fig. 2C). Marked changes in the degree to which burst responses facilitate within a theta train can affect the size and stability of LTP. We tested for differences in within-train facilitation by expressing the composite areas of burst responses 2-10, respectively, as a fraction of the area of burst response 1 . Both age groups showed within-train facilitation, but this was much more pronounced for the slices from the younger rats. As shown in Figure $2 D$, the second burst response in the young adults was $78 \pm 10 \%$ larger than the first burst response; the equivalent change for the middle-aged slices was only $43 \pm 5 \%$. This dramatic difference in burst facilitation was maintained throughout the theta train (two-way repeated-measures ANOVA; $p<0.001$ ).

In marked contrast to the above results, age-related differ- 
A.

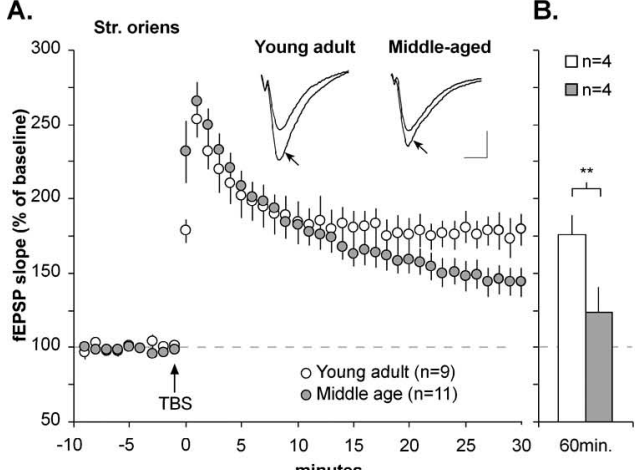

E.

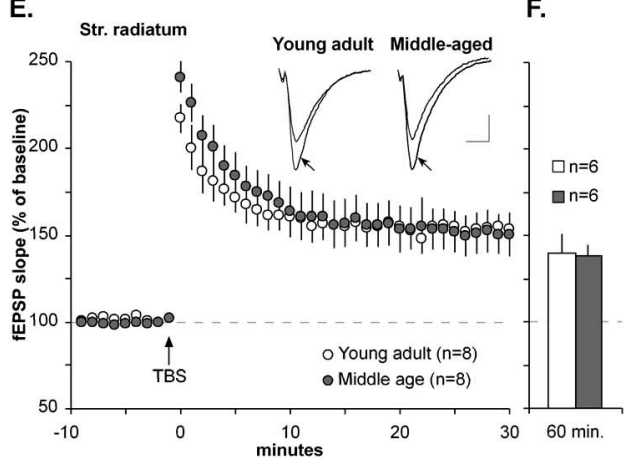

C.

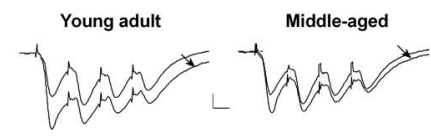

D.

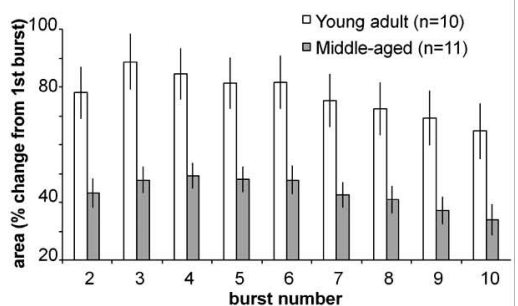

G.

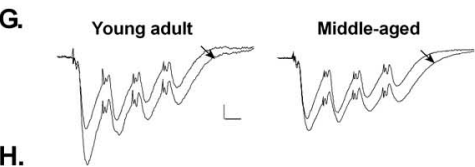

H.

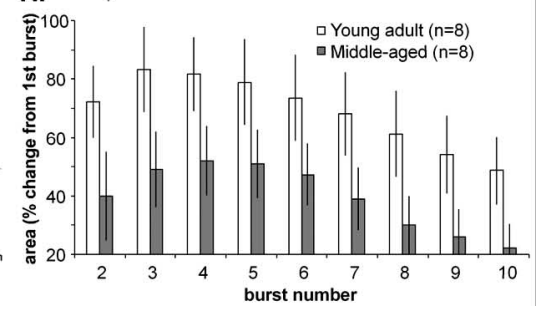

Figure 2. LTP fails to stabilize in basal, but not apical, dendrites from 7- to 10-month-old rats. $\boldsymbol{A}$, Plot of group data for the Schaffer-commissural fEPSP slope within the basal dendritic field of hippocampal slices from young adult (1-2 months of age) and middle-aged ( $8-10$ months of age) rats before and after LTP induction. TBS was applied to induce LTP after a 10 min baseline at the time indicated by an arrow. As shown, middle-aged slices showed no deficits in the magnitude of LTP within the first 10 min after a train of 10 theta bursts but deviated from the young adult pattern by continuing to decay thereafter. Inset, Representative traces recorded from the str. oriens during the baseline recording period and $30 \mathrm{~min}$ after TBS (arrow). Calibration: $0.5 \mathrm{mV}, 10 \mathrm{~ms}$. $B$, Slope of the fEPSP in a subset of young adult and middle-aged slices $60 \mathrm{~min}$ after TBS; values indicate that LTP remained stable in young adult slices but continued to decline in the middle-aged group $\left({ }^{* *} p<0.01\right.$; two-tailed $t$ test). $C$, Burst responses recorded during TBS. Averaged responses (from a subset of 4 rats) from burst 1 and burst 4 (arrow) are superimposed. Facilitation from burst 1 to burst 4 is notably smaller in slices from middle-aged rats compared with young adults. Calibration: $0.5 \mathrm{mV}, 10 \mathrm{~ms}$. D. Comparison of the size of theta-burst responses within a theta train in young adult and middle-aged rats: the plot shows the mean change in the area of responses $2-10$, relative to the first theta-burst response; the middle-aged slices had much smaller within-train facilitation (average area of bursts $2-10,43 \pm 4.7 \%$ ) compared with slices from young adults (77 $\pm 8.6 \%$ ). $\boldsymbol{E}$, Plot of group data showing the Schaffer collateral/commissural fEPSP slope within the str. radiatum of young adult and middle-aged slices before and after TBS. As is apparent, the fEPSP slopes in both groups were comparable across the $30 \mathrm{~min}$ testing period after TBS. Inset, Representative fEPSPs recorded from the str. radiatum in slices from young adult and middle-aged rats during the baseline recording period and $30 \mathrm{~min}$ after TBS (arrow). Calibration: $0.5 \mathrm{mV}, 10 \mathrm{~ms}$. $\boldsymbol{F}$, Potentiation in the apical dendrites of young adult and middle-aged slices was equivalent 60 min after TBS. G, Averaged responses (from a subset of 4 rats) during burst 1 and burst 4 (arrow) are overlaid for young adult and middle-aged rats. Note that the middle-aged group exhibited reduced facilitation in the apical dendrites, as was the case for the basal dendrites. Calibration bars: $0.5 \mathrm{mV}, 10 \mathrm{~ms}$. $\boldsymbol{H}$, Comparison of the size of theta-burst responses within a TBS train in young and middle-aged rats: the plot shows the mean change in the area of burst responses $2-10$ relative to the size of the first theta-burst response. Slices prepared from middle-aged rats had significantly smaller within-train facilitation in the apical dendrites than did those from young adults.

ences in LTP were not evident in the apical dendrites. As shown in Figure $2 E$, there was a large initial increase in fEPSP slope at $1 \mathrm{~min}$ after induction in young adult $(100 \pm 11 \%$ above baseline) and middle-aged tissue $(126 \pm 13 \%)$ that was followed by a decay period lasting for $\sim 15 \mathrm{~min}$, after which response size reached a plateau at $55 \pm 14 \%$ above baseline in the young adult and $57 \pm$ $8 \%$ in the middle-aged slices. LTP then persisted throughout the remainder of the 60 min testing period (Fig. $2 F$ ), with no evident differences between the two groups (two-way repeated-measures ANOVA; $p>0.3$ for minutes 50-60). Note that the magnitude of LTP in the apical dendrites is comparable with that typically reported for the region in field potential studies (Arai et al., 1994; Kramár and Lynch, 2003) and, again in agreement with previous reports (Capocchi et al., 1992; Arai et al., 1994; Leung and Shen,
1999; Kramár and Lynch, 2003), is substantially smaller than LTP within the str. oriens (Fig. 2, compare $A, E$ ).

Comparisons of burst response facilitation in the apical dendrites are described in Figure 2, $G$ and $H$. Unexpectedly, facilitation again proved to be substantially depressed in the middle-aged group relative to the young adults. This result indicates that aging acts broadly across the CA1 region during the early stages of adulthood to reduce burst response facilitation in both apical and basal dendritic fields and that this depression by itself is not sufficient to substantially affect the magnitude of LTP in middle-aged slices. It follows from this second point that there is a considerable range of theta-burst sizes (as seen in young adult vs middle-aged apical dendrites) over which the LTP stabilization process can be triggered. Two questions then arise: (1) what causes the generalized depression of theta-burst facilitation in field CA1 of the older slices, and (2) what is responsible for the loss of LTP consolidation in the basal dendrites?

\section{Enhancing theta bursts overcomes impairment to LTP consolidation}

Adenosine is released by afferent activity and depresses synaptic responses, in part via presynaptic $\mathrm{A}_{1} \mathrm{Rs}$ (Dunwiddie and Haas, 1985). Accumulation of the nucleoside during theta stimulation could, therefore, counteract within-train facilitation of burst responses. We tested the effects of blocking $A_{1}$ Rs on the impaired facilitation of burst responses in middle-aged slices using the potent antagonist DPCPX. During drug infusion, the antagonist increased the str. oriens fEPSP by $22 \pm 9 \%$ in young adult slices and by $26 \pm 7 \%$ in the middleaged cases. As described in the literature for apical dendrites (Arai and Lynch, 1992; Costenla et al., 1999), the increased responses were accompanied by a decrease in paired-pulse facilitation (50 ms interval) in both middle-aged $(-18 \pm 6 \%)$ and young adult $(-23 \pm 8 \%)$ slices $(p>0.4$; two-tailed $t$ test). After drug wash-in, stimulus intensity was reduced to reset fEPSP amplitudes back to baseline levels. New baselines were established after responses reached stable values and theta bursts were delivered; LTP was measured and expressed as a percentage of the DPCPX baseline. The drug was present throughout the remainder of the experiment. Figure $3 \mathrm{~A}$ summarizes the magnitude of burst facilitation recorded in the presence of DPCPX from a group of middle-aged slices; for comparison purposes, the results are shown together with those obtained from middle-aged slices in the absence of the drug (Fig. 2C). With DPCPX treatment, the second burst was increased over the first by $80 \pm 9 \%$ (Fig. $3 A$ ), a value comparable with that described previously for young adults under control conditions. Moreover, the mean facilitation over burst 1 for bursts $2-10$ (80 $\pm 9 \%)$ was 
A.

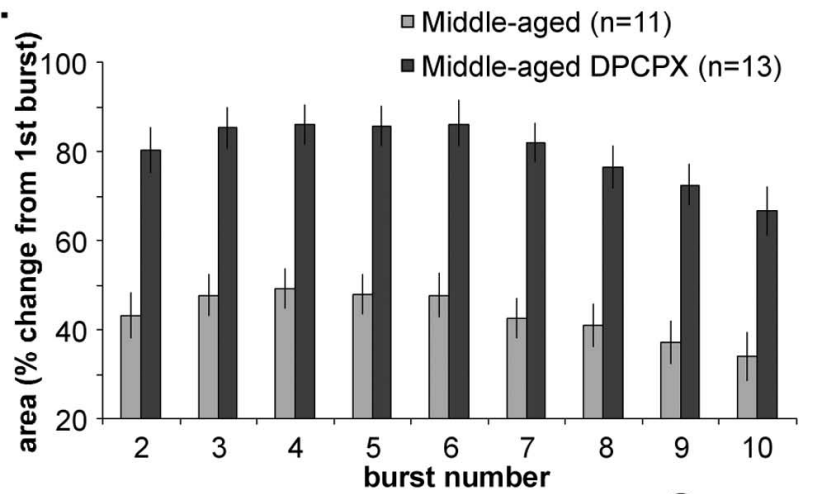

B.

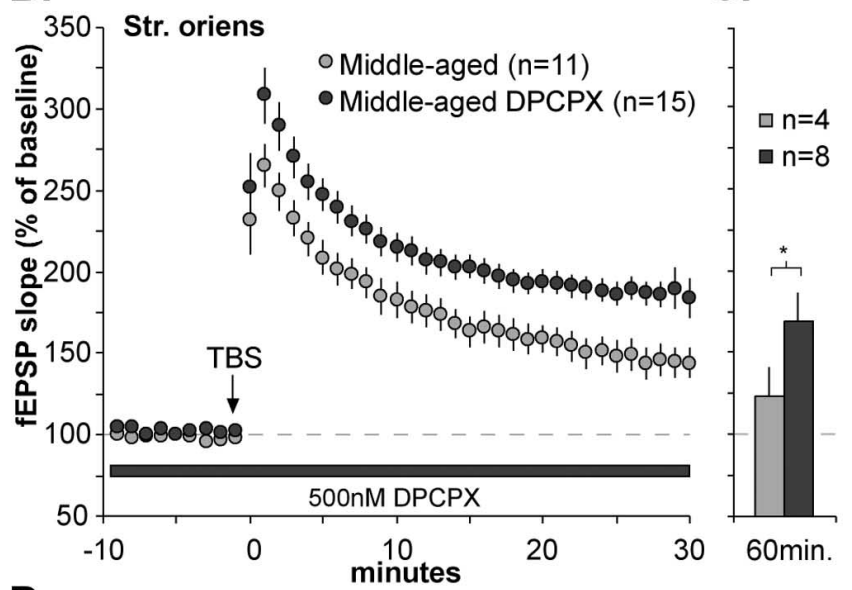

D.

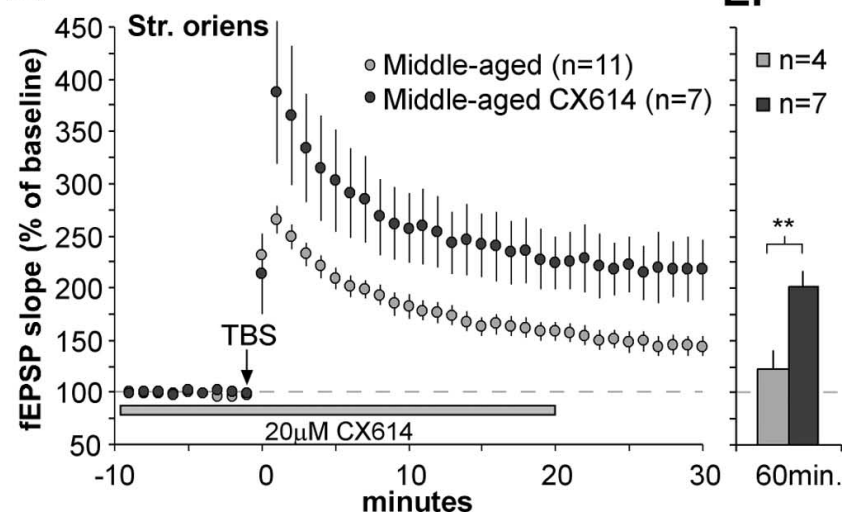

Figure 3. The adenosine $A_{1}$ antagonist DPCPX and an AMPA receptor-positive modulator rescue LTP stabilization in middle-aged basal dendrites. The effects of TBS on Schaffercommissural fEPSPs within the stratum oriens were evaluated in slices from middle-aged rats with and without $500 \mathrm{~nm}$ DPCPX treatment. $\boldsymbol{A}$, Comparison of the composite area of burst responses within a theta train in slices from middle-aged rats with and without DPCPX. The graph summarizes the mean percentage change in the area of burst responses $2-10$ relative to the size of the first burst response. DPCPX significantly increased theta train facilitation, bringing it into the range of facilitation levels seen in young adult tissue (compare with Fig. 2D). $\boldsymbol{B}$, Plot of fEPSP slope before and after TBS (values normalized to the mean response during the last 10 min of the baseline period in the presence of DPCPX). As shown, DPCPX treatment enhanced the magnitude of LTP in middle-aged slices at all time points. C, DPCPX-treated slices from middle-aged animals tested for $1 \mathrm{~h}$ after TBS showed stable potentiation that was significantly enhanced compared with middle-aged control slices ( ${ }^{*} p<0.05$; two-tailed $t$ test). $\boldsymbol{D}$, A positive modulator of AMPA receptors also enhances $L T P$ in the basal dendrites of middle-aged rats. The percentage of LTP induced by a single train of theta bursts was measured in the stratum oriens in the presence and absence of $\mathrm{CX614}(20 \mu \mathrm{M})$. The graph of fEPSP slopes is expressed as percentage of the mean baseline response during the 10 min preceding TBS in the presence of CX614; slices infused with CX614 had greater LTP during the 30 min after TBS. $E$, The positive effects of $C X 614$ were maintained throughout the 60 min after TBS. $\left({ }^{* *} p<0.01\right.$ compared with drug-control values; two-tailed $t$ test). also about the same as that obtained for young adults under control conditions (78 $\pm 10 \%$; two-way repeated-measures ANOVA; $p>0.8$ ) and was significantly greater than that obtained in the str. oriens for middle-aged slices without DPCPX treatment $(43 \pm 5 \% ; p<0.01)$. Thus, DPCPX appears to fully reverse age-related losses in within-train facilitation. As described previously for apical dendrites (Arai and Lynch, 1992), young adult rats did not show a significant enhancement of burst facilitation with $A_{1}$ antagonist treatment (data not shown).

The DPCPX-associated changes in the characteristics of TBS train responses were accompanied by a substantial increase in the magnitude of LTP in middle-aged slices. Potentiation in slices treated with DPCPX decayed for the first 15 min after TBS but then appeared to stabilize at a value well above that seen in control (non-DPCPX treated) middle-aged slices (Fig. 3B). The percentage of LTP at the time point $30 \mathrm{~min}$ after TBS was $85 \pm 7 \%$ above baseline in the presence of DPCPX, a value that was both substantially greater than in middle-aged slices without the antagonist present $(44 \pm 10 \% ; p<0.01$; two-tailed $t$ test) and about the same as that recorded for the young adult slices $(p>0.4)$. Tests in a subgroup of eight middle-aged slices (Fig. $3 C$ ) showed that enhanced LTP persisted for at least $1 \mathrm{~h}$ after TBS in the presence of DPCPX $(p<0.01$; two-way repeated-measures ANOVA for minutes 50-60). Previous studies of apical dendrites in slices from young adult rats indicate that DPCPX, although it increases the degree of potentiation elicited by a suboptimal number of theta bursts, has no effect on the percentage of LTP induced by a conventional train of 10 bursts (Arai et al., 1990b). This was confirmed in the present study (mean facilitation for young adult DPCPX-treated slices; $88 \pm 16 \%$; $p>0.2$; two-way repeated-measures ANOVA; $n=3$ ). Thus, DPCPX eliminates much, if not all, of the difference between young and middle-aged rats, rather than simply amplifying potentiation at both ages.

The results obtained with DPCPX indicate that the basal dendrites in middle-aged rats are competent with regard to the expression of LTP but, in the absence of the antagonist, apparently fail to engage processes that stabilize potentiation. DPCPX could restore consolidation by (1) increasing the magnitude of the induction event and thereby overcoming the block, (2) reducing the block itself, or (3) both. To test the plausibility of the first of these ideas, we used a manipulation unrelated to adenosine that enhances the magnitude of theta-burst responses. The ampakine CX614 is one of a family of centrally active, positive modulators of AMPA-type glutamate receptors (Lynch, 2002) that have been shown to increase responses to theta bursts (Arai et al., 2004). Figure $3, D$ and $E$, summarizes the results from a set of experiments in which CX614 was applied to slices throughout the baseline and LTP periods, following a protocol similar to that used for DPCPX. As shown, LTP in the middle-aged slices, when induced in the presence of the ampakine, had the same size and stability as LTP in young adults. These results indicate that simply increasing the size of theta bursts overcomes whatever factors are responsible for impaired consolidation in the basal dendrites of middleaged slices.

\section{Adenosine does not block theta-burst facilitation but does prevent consolidation}

The results obtained with the $A_{1} R$ antagonist DPCPX suggested that build-up of adenosine during the theta train depresses either theta-burst responses or the processes responsible for their facilitation during the train. To discriminate between these possibilities, we measured the effects of exogenous adenosine on burst size and facilitation in the basal dendrites of young adult slices. 
A.

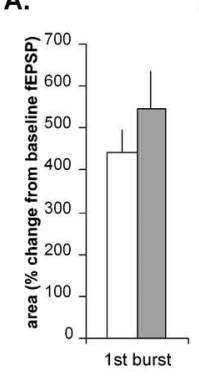

B.

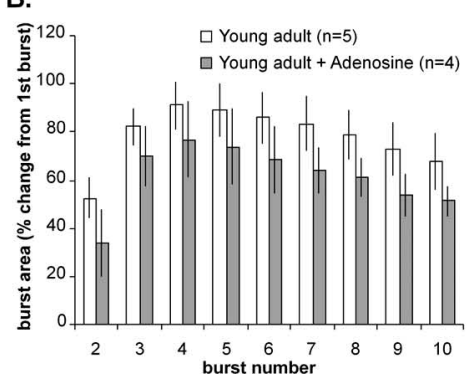

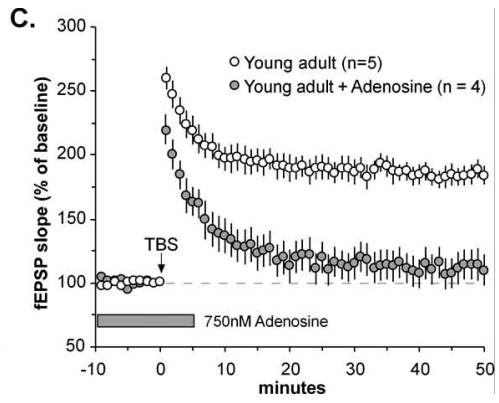

Figure 4. Low concentrations of adenosine do not block theta-burst facilitation but do prevent LTP consolidation in the stratum oriens of young adult slices. $\boldsymbol{A}$, The areas of single theta-burst responses (i.e., the composite potential generated by 4 pulses at $100 \mathrm{~Hz}$ ) are expressed as a fraction of baseline fEPSP area (during the 5 min preceding TBS). Group values show that the burst area is increased over the fEPSP area to the same degree in adenosine-treated (gray bar) and control (white bar) slices. $\boldsymbol{B}$, The plot shows the mean change in the size of burst responses $2-10$, relative to the size of the first theta-burst response within a theta train; facilitation of burst responses was not substantially affected by infusion of $750 \mathrm{~nm}$ adenosine. C, A plot showing the effects of TBS on Schaffer-commissural fEPSP slopes with and without bath adenosine treatment in the basal dendritic field of young adult slices. Adenosine was added to perfusion lines at $750 \mathrm{~nm}$ for $40 \mathrm{~min}$ before the delivery of TBS and washed out 5 min afterward. As is apparent, exogenous adenosine completely suppressed the stabilization of LTP (2-way repeated-measures ANOVA; $p<0.01$ ).

A.

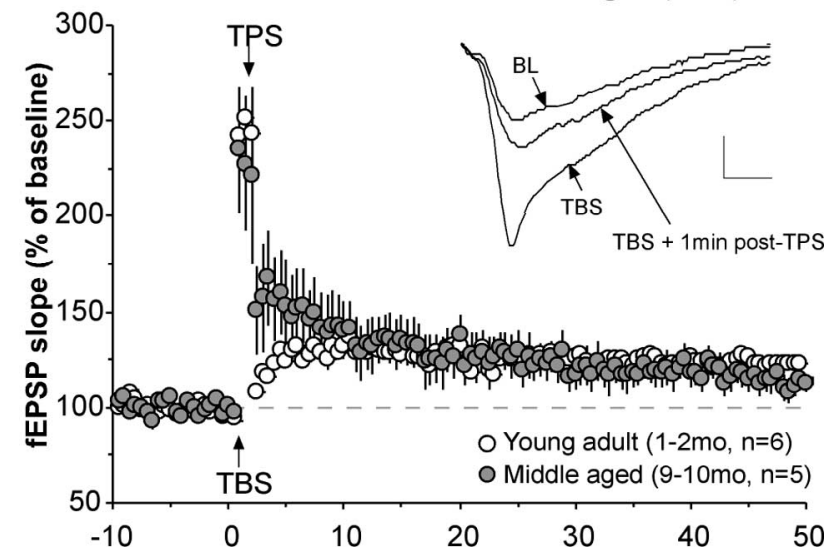

Figure 5. The LTP reversal effect is fully operational in the basal dendrites of middle-aged slices. TBS was applied to Schaffer-commissural projections in the stratum oriens in slices prepared from young adult (1-2 months of age) or middle-aged ( $9-10$ months of age) rats. $\boldsymbol{A}, \boldsymbol{B}$, A single train of $5 \mathrm{~Hz}$ stimulation (TPS) was delivered to the potentiated pathway beginning $60 \mathrm{~s}$ after TBS. $\boldsymbol{A}$, The graph summarizes group results and confirms that potentiation did not recover but instead decayed almost to baseline over the following $30 \mathrm{~min}$. $\boldsymbol{B}$, As seen in the traces from a representative experiment, the $5 \mathrm{~Hz}$ stimulation caused an immediate and pronounced loss of potentiation in middle-aged slices.

Adenosine at $750 \mathrm{~nm}$ reduced the size of field EPSPs by $16 \pm 8 \%$ within $20 \mathrm{~min}$ of adding it to the infusion line. Recordings were then continued for an additional 20 min to establish a new baseline, after which theta bursts were applied. As shown in Figure $4 A$, the area of the first theta-burst response was $548 \pm 77 \%$ of the size of baseline field EPSPs (mean of responses during the 5 min preceding TBS); this is comparable with values in young adult control slices ( $443 \pm 53 \%$; two-tailed $t$ test; $p>0.3$ ). Because baseline fEPSPs were smaller after adenosine treatment, it follows that theta responses would also be smaller than under control conditions. Yet, burst facilitation during the train was equivalent under adenosine treatment and control conditions (Fig. $4 B$ ); in the presence of adenosine, burst responses 2-10 were, on average, $61 \pm 12 \%$ greater than the first burst response, whereas the comparable control value was $78 \pm 10 \%$ (two-way repeated-measures ANOVA; $p>0.3$ ). Together, these results favor the argument that adenosine build-up minimally influ-

ences the within-train facilitation process itself but instead depresses individual fEPSPs and hence the size of their composite response to short bursts of afferent stimulation.

Although adenosine had little effect on the initial fEPSP potentiation (Fig. 4C) elicited by theta stimulation (1 min after TBS, $225 \pm 8$ vs $263 \pm 10 \%$ above baseline for adenosine-treated and controls, respectively; $p>0.05$; two-tailed $t$ test), it caused a near complete block of LTP consolidation ( $p<0.01$, two-way repeatedmeasures ANOVA for minutes 40-50 after TBS). It should be noted that the burst responses 2-10 recorded in the presence of adenosine were only slightly smaller than those found in control young adult slices and were considerably larger than those in middle-aged slices. This suggests that the near complete block of stabilization produced by adenosine was attributable to factors other than a reduction in the initial triggering event for LTP (i.e., AMPA receptor-mediated depolarization) (for review, see Nicoll, 2003); that potentiation was not significantly different from control immediately after the train also points to this conclusion.

\section{Blocking adenosine $A_{1}$ Rs after theta-burst stimulation restores LTP consolidation}

An alternative possibility to the one postulated above is that the age-dependent loss of LTP consolidation is attributable to the activation of the previously described LTP reversal process by adenosine. It has been repeatedly demonstrated that the reversal effect is blocked by $\mathrm{A}_{1} \mathrm{R}$ antagonists, even when applied after LTP induction (Larson et al., 1993; Staubli and Chun, 1996; Fujii et al., 1997; Huang et al., 1999; Omrani and Fathollahi, 2003). This explanation could also account, at least in part, for the restoration of LTP consolidation in middle-aged slices by DPCPX. The argument, however, requires that reversal mechanisms be preserved in the basal dendrites of middle-aged rats. We tested this point and found that a single $1 \mathrm{~min}$ train of $5 \mathrm{~Hz}$ stimulation [theta pulse stimulation (TPS)] applied 1 min after LTP induction in the basal dendrites of middle-aged slices caused potentiation to immediately drop from a value of $131 \pm 23$ to $54 \pm 20 \%$ above baseline (Fig. 5A, B). At $30 \mathrm{~min}$ after TBS (i.e., $29 \mathrm{~min}$ after TPS), potentiation in middle-aged slices $(21 \pm 8 \%)$ was similar to postreversal potentiation in young adults $(26 \pm 11 \%)$ but was significantly lower than values in age-matched slices that did not receive TPS ( $44 \pm 10 \% ; p<0.05$; two-tailed $t$ test) (Fig. $2 A$ ).

The above results establish that the LTP reversal effect is fully functional in the str. oriens of middle-aged slices, making it possible that excessive build-up of adenosine during theta trains uses this mechanism to disrupt LTP consolidation in middle-aged slices. If so, infusion of DPCPX immediately after theta-burst stimulation should fully restore potentiation in these slices. This was tested by lowering a DPCPX-filled micropipette $(2.5 \mu \mathrm{M})$ into the chamber next to the slice and then starting infusion within $15 \mathrm{~s}$ of the termination of a theta-burst train. The infusion was continued for only $3 \mathrm{~min}$, after which slice conditions were returned to normal (i.e., the infusion was stopped and the DPCPX micropipette was removed from the chamber). All experiments were performed using a two-input stimulation para- 
digm in which one of two converging collections of basal Schaffer-commissural projections received the theta train, whereas the second served as control pathway and as an indicator that the DPCPX, after its dilution after leaving the infusion pipette, reached the target synapses in physiologically effective concentrations (i.e., that it transiently increased the control fEPSP). Figure 6 summarizes the results. DPCPX increased the size of the control response within $30 \mathrm{~s}$ of the onset of pipette infusion, and this effect was reversed within 2-3 min of terminating the treatment (Fig. 6B). Slices treated with control aCSF in the micropipette did not show this effect, and their experimental (TBS) pathways exhibited the same decremental LTP described above. In contrast, slices treated with DPCPX (Fig. 6A) showed augmented responses immediately after the theta train, presumably because of enhanced release from the DPCPX and had robust LTP that persisted for the full $50 \mathrm{~min}$ of post-TBS recording (50 min after TBS, $87 \pm 23$ vs $20 \pm 12 \%$ above baseline for DPCPX and vehicle controls, respectively; $p<0.01$; two-way repeated-measures ANOVA for minutes 40-50).

DPCPX is reported in the literature (Arai et al., 1990b) not to enhance the magnitude of LTP produced by a fulllength theta-burst train in hippocampus of young adult slices, and, as discussed above, we obtained results that were in agreement with this. The point also held with the postinduction treatment paradigm, in that a 3 min infusion of DPCPX immediately after theta-burst stimulation in the str. oriens of young adult slices did not increase potentiation above that seen in untreated slices ( $50 \mathrm{~min}$ after TBS, $85 \pm$ 26 vs $77 \pm 22 \%$ for DPCPX and vehicle controls, respectively; $p>0.4$; two-way repeated-measures ANOVA; $n=2$ for both groups). It is still possible that a stimulation paradigm of 10 theta bursts produced LTP in young adult slices that was near its ceiling and consequently insensitive to any DPCPX-mediated enhancements of LTP. Therefore, we repeated the experiment using a suboptimal amount of stimulation (five theta bursts) delivered to str. oriens of young adult slices. Figure $6, C$ and $D$, illustrates the effects of a 3 min application of DPCPX immediately after this abbreviated train. DPCPX applied immediately after induction again did not increase potentiation ( $p>0.5$; two-way repeated-measures ANOVA for minutes 40-50) in treated (50 min after TBS, $29 \pm$ $14 \%)$ versus vehicle-treated controls $(21 \pm 5 \%)$. It thus appears that endogenous adenosine, in the immediate post-theta-burst period, blocks consolidation only in the basal dendrites of older slices. lated control pathway.
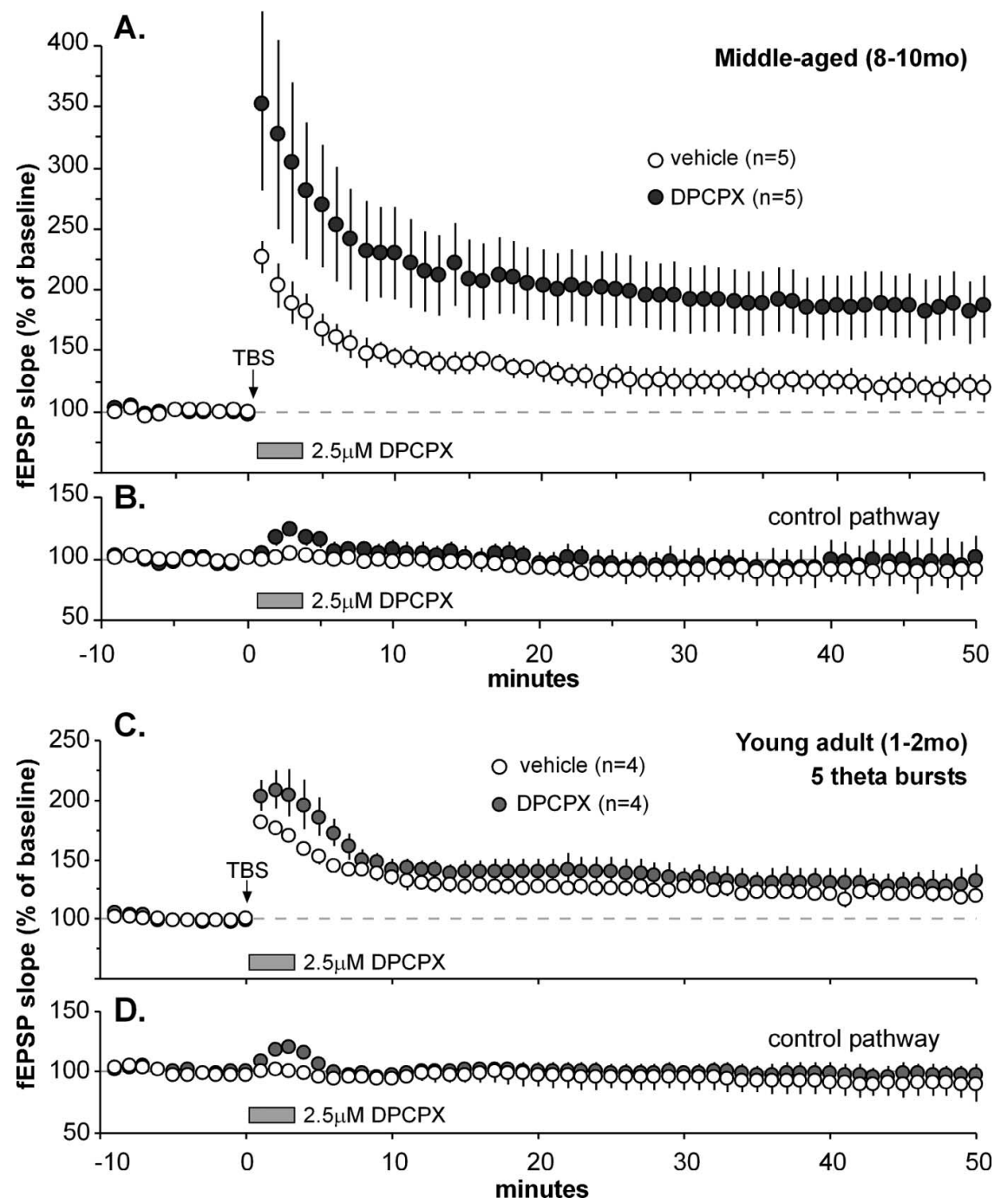

Figure 6. Adenosine $A_{1}$ receptor antagonist applied after TBS rescues robust LTP in middle-aged slices. TBS was applied to the Schaffer-commissural system in the str. oriens, immediately after which the $A_{1}$ receptor antagonist DPCPX or vehicle was infused from an adjacent micropipette controlled via an automated pump. fEPSPs were simultaneously recorded from a second collection of Schaffer-commissural projections (the "control pathway") to confirm that the drug was working in the DPCPX cases and to ensure that the infusion itself did not disrupt baseline recording in the control (vehicle) cases. $A$, Slices in which TBS was followed by vehicle infusion (open circles) exhibited an LTP effect that did not stabilize but instead decayed almost to baseline during the postinduction period (baseline indicated by dashed line). In contrast, a 3 min infusion of DPCPX immediately after TBS (filled circles) caused potentiation to stabilize at $83 \pm 24.7 \%$ above baseline (i.e., values comparable with those found in young adult slices) at 50 min after induction. $\boldsymbol{B}$, Effects of vehicle or DPCPX infusions on the converging Schaffer-commissural control pathway that did not receive TBS. As shown, slices treated with DPCPX, but not vehicle, exhibited a brief response facilitation, indicating that the $A_{1} R$ antagonist quickly reached the recording sites at effective concentrations and subsequently washed out with little delay. C, Suboptimal stimulation (5 theta bursts) was delivered to the str. oriens of young adult slices, followed immediately by a 3 min infusion of DPCPX (filled circles) or vehicle (open circles). By 50 min after stimulation, DPCPX-treated slices did not show greater potentiation than vehicle-treated slices ( $p>0.5 ; 2$-way repeated-measures ANOVA for minutes $40-50$ ), indicating that blocking $A_{1}$ receptors after stimulation does not increase maximum LTP. $D$, Effects of vehicle or DPCPX infusions on the nonstimu-

Adenosine receptors in young and middle-aged slices

The results described in the preceding section suggest that $A_{1} R s$, or the processes they trigger, undergo age-related changes that disrupt LTP in the basal dendrites of field CA1. We explored this idea by comparing the degree to which brief infusions of adenosine depressed synaptic responses in the apical and basal dendrites of young and middle-aged slices. Figure 7 summarizes results for experiments in which various concentrations of the ligand were added to the infusion lines for $5 \mathrm{~min}$, and responses were simultaneously collected in the apical and basal dendritic 

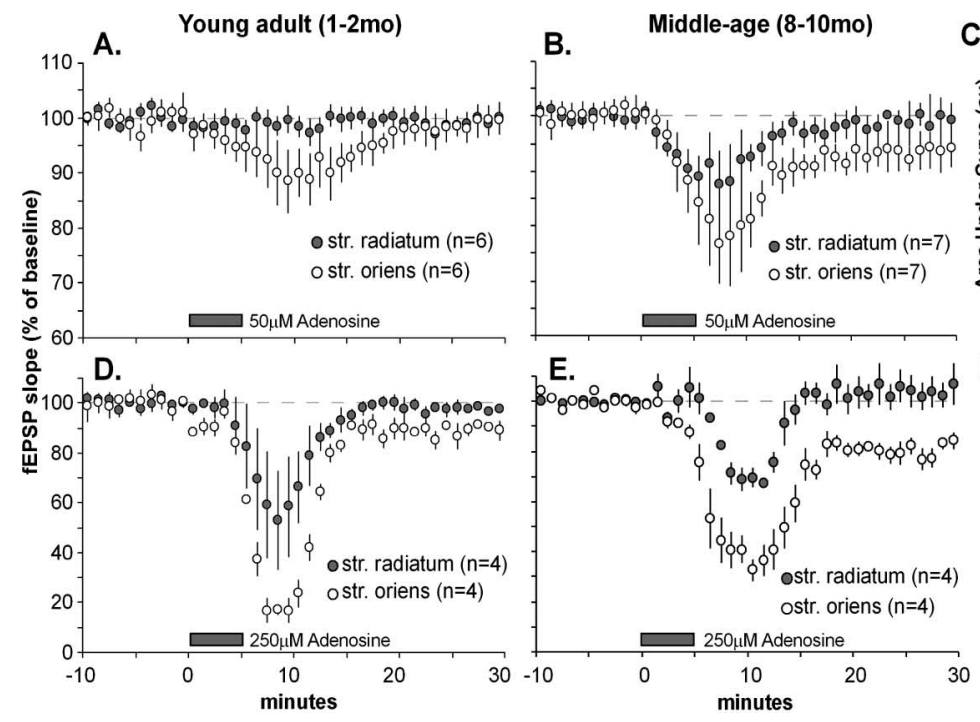

Figure 7. Region- and age-related differences in adenosine effects on synaptic responses. High concentrations of adenosine were added to perfusion lines for $5 \mathrm{~min}$ and then washed out for $25 \mathrm{~min}$. fEPSPs generated by stimulation of the apical and basal branches of the Schaffer-collateral/commissural projections were recorded simultaneously in the str. radiatum and str. oriens, respectively. $\boldsymbol{A}, \boldsymbol{B}$, Adenosine at $50 \mu \mathrm{m}$ caused a rapid reduction of fEPSP slope that was greater in the str. oriens than in the str. radiatum in both young adult $(\boldsymbol{A})$ and middle-aged $(\boldsymbol{B})$ slices. $\boldsymbol{C}$, Quantification of the area under the curve (i.e., the deviation from baseline as plotted in $\boldsymbol{A}$ and $\boldsymbol{B}$ and expressed in arbitrary units) from the time of initial infusion of $50 \mu \mathrm{m}$ adenosine to 15 min after drug washout. Responses for the str. radiatum (SR) and str. oriens (SO) show age-related differences in drug sensitivity in both strata ( $p>0.05$; two-tailed $t$ test). $\boldsymbol{D}, \boldsymbol{E}$, High concentrations of adenosine ( $250 \mu \mathrm{m})$ markedly depressed Schaffer-collateral/ commissural fEPSPs with the same pattern of regional differences as that seen at the lower concentration. Note that washout was not completely effective in the str. oriens of the middle-aged slices. $\boldsymbol{F}$, Dose-response relationships using area under the curve as a response measure. Differences were not noted between young adult (YA; open symbols) and middle-aged (MA; filled symbols) slices with high-concentration adenosine infusions (250 and $500 \mu \mathrm{m}$ ), but striking differences between SO (circles) and SR (diamonds) were observed for both age groups.

fields. Adenosine at $50 \mu \mathrm{M}$ caused a very rapid reduction of fEPSP slope, and this effect was greater in the basal than in the apical dendrites in both young (Fig. 7A) and middle-aged slices (Fig. $7 B$ ). Adenosine was also more potent in both dendritic domains in middle-aged slices than in slices from young adults. A comparison of the total response depression during the period of adenosine treatment (i.e., the area under the curve from the beginning of drug infusion until $15 \mathrm{~min}$ after the start of washout) indicated that the middle-aged slices were significantly more sensitive to adenosine than the young adult slices (Fig. $7 C)(p<0.05$; twotailed $t$ test). This effect did not hold at higher concentrations; the effects of a $5 \mathrm{~min}$ infusion of $250 \mu \mathrm{M}$ adenosine were as great, or greater, in the young slices (Fig. 7D) than in the middle-aged cases (Fig. 7E). Note, however, that the differential potency of adenosine on simultaneously recorded apical versus basal responses seen at the lower concentration was still in evidence. Figure $7 F$ summarizes the regional difference in potency across the two concentrations and indicates that the young versus middle-age differences were present only at the lowest concentration tested.

Washout of adenosine even at $250 \mu \mathrm{M}$ was very rapid in the str. radiatum, as might be expected given that very short infusions were used. Interestingly, washout appeared to be less complete in the str. oriens, particularly in the older slices (Fig. $7 B, D$ ). This effect in middle-aged slices was of borderline significance at 50 $\mu \mathrm{M}$ (two-way repeated-measures ANOVA; $p<0.1$ ) and highly significant at $250 \mu \mathrm{M}(p<0.01)$.

The above results raise the question of whether levels of $A_{1}$ Rs (1) differ between apical and basal dendrites and/or (2) change with entry into middle age. Because these issues appear not to
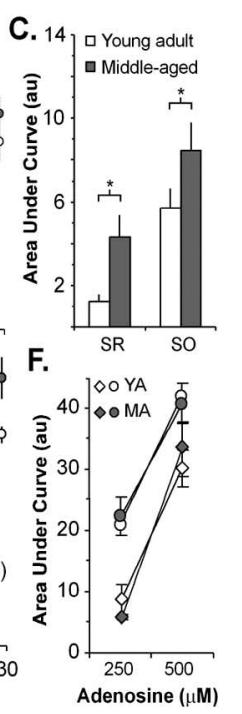

have been addressed in the literature, we performed an immunocytochemical analysis of $A_{1} R$ levels in the hippocampus of 2and 9-month-old rats. Photomicrographs of representative sections are presented in Figure $8, A$ and $B$. The cell body layers throughout hippocampus exhibited low levels of immunoreactivity, as did stratum lacunosum-moleculare of field CA1. Stratum lucidum and the hilus of the dentate gyrus, areas containing the mossy fibers, were detectably more lightly stained than neighboring dendritic zones. In contrast, strata radiatum and oriens of the hippocampus proper, both zones occupied by the terminals of hippocampal commissural and associational axonal systems, contained high levels of immunoreactivity, as did the stratum moleculare of dentate gyrus. Some differentiation was apparent within these areas and, in particular, immunostaining was less dense in the apical dendritic layers of CA3 and dentate gyrus than it was in the dendritic fields of CA1. These results are in general agreement with previous studies of ligand binding (Lee et al., 1986; Swanson et al., 1995; Shearman and Weaver, 1997) and, in fewer instances, immunostaining (Rivkees et al., 1995; Swanson et al., 1995) for adenosine $\mathrm{A}_{1}$ Rs.

Quantitative tests for age-related differences in $A_{1} R$ levels were performed by dissecting the two laminas from $350-\mu$ m-thick vibratome sections through the contralateral hippocampus from the same young (1-2 months of age) and middle-aged (8-10 months of age) rats used for electrophysiological analyses. Laminar samples from individual rats were run for Western blot analysis of $A_{1} R$ immunoreactivity (Fig. $8 C$ ). As shown in Figure $8 D$, there were no significant differences in levels of $A_{1} R$ immunoreactivity between the str. oriens and str. radiatum in young or middle-aged rats, and, furthermore, there were no significant differences between age groups for $\mathrm{A}_{1} \mathrm{R}$ levels in the str. oriens ( $p>0.2$; twotailed $t$ test) or str. radiatum $(p>0.9)$. A constant level of $A_{1} R s$ could translate into greater potency if the number of excitatory synapses in the str. oriens were to decrease with age. To test for this, we measured levels of immunoreactivity for the glutamate receptor scaffolding protein PSD-95, a marker expected to be sensitive to changes in synaptic numbers (Sans et al., 2000). PSD-95 levels were similar in the str. oriens and str. radiatum and in young versus middle-aged animals (Fig. $8 E$ ). In all, these results indicate that differences in $\mathrm{A}_{1}$ adenosine receptor densities are not likely to account for regional differences or age-related changes in the sensitivity of synapses to adenosine or the stability of LTP.

\section{Discussion}

The above results describe two novel aspects of age-related changes in synaptic plasticity: (1) the negative effects of aging on LTP manifest themselves much earlier than had been suspected previously, and (2) aging acts differentially across the dendritic domains of a single population of neurons. Deficits in CA1 apical dendritic LTP have been reported for aged rats and mice (Deu- 

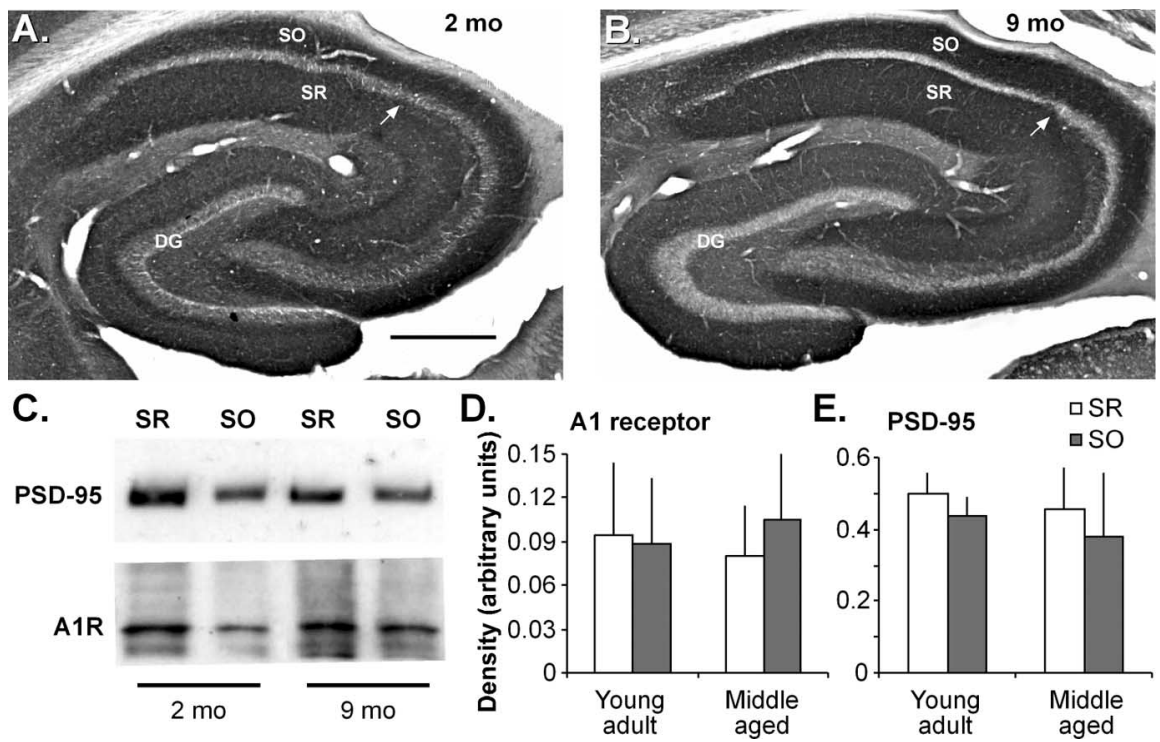

E. PSD-95 aSO

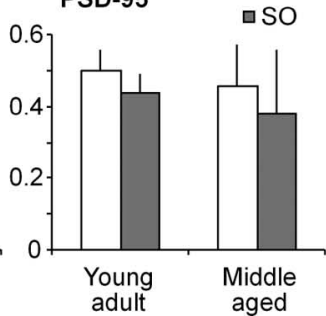

Figure 8. Regional adenosine $A_{1}$ receptor levels in the hippocampus of rats of different ages. $A, B$, Low-magnification photomicrographs of immunostaining for the $A_{1}$ receptor in sections through the hippocampus of 2-month-old $(\boldsymbol{A})$ and 9 -month-old $(B)$ rats shows comparably dense immunostaining in the CA1 str. oriens (SO) and str. radiatum (SR). The arrow indicates the approximate CA1/CA2 boundary. Scale bar: (in $A) A, B, 500 \mu \mathrm{m}$. C, Representative Western blot shows levels of PSD-95 (top) and $A_{1}$ receptor (A1R; bottom) immunoreactivities in homogenates from the $C A 1$ str. radiatum (SR) and str. oriens (SO) from young adult (1-2 months of age) and middle-aged ( $8-10$ months of age) rats (10 $\mu \mathrm{g}$ of protein per lane; immunoreactive bands were 34 and $89 \mathrm{kDa}$, respectively). $\boldsymbol{D}, \boldsymbol{E}$, Graphs show levels of $\mathrm{A}_{1}$ receptor $(\boldsymbol{D})$ and PSD-95 $(\boldsymbol{E})$ immunoreactivities assessed by densitometric analysis of Western blot ECL films ( $n=6$ rats/age).

pree et al., 1993; Moore et al., 1993; Shankar et al., 1998), although other authors found normal-appearing potentiation in such animals (Landfield and Lynch, 1977; Landfield et al., 1978; Applegate and Landfield, 1988; Chang et al., 1991; Barnes et al., 1996; Norris et al., 1996). Stimulation paradigm appears to be one factor determining whether deficits are seen (Lanahan et al., 1997; Tombaugh et al., 2002; Watabe and O’Dell, 2003), suggesting that triggering, rather than expression, mechanisms for LTP are vulnerable to old age. The present findings indicate that deficits in LTP emerge well in advance of old age and are present in basal dendritic fields of middle-aged rat hippocampus. There appear to be no precedents for the observed differential effects of aging between apical and basal dendritic arbors.

Results described here also provide the first evidence that synaptic responses to trains of theta-burst stimulation undergo pronounced changes in the transition from young adulthood to middle age. Experiments using DPCPX indicated that adenosine $A_{1}$ receptors are involved in both LTP and theta train deficits but most likely through different signaling cascades. The antagonist eliminated age differences in theta-burst facilitation, suggesting that adenosine build-up during the train either (1) disrupts the facilitation process itself or (2) simply depresses release and thereby offsets the effects of facilitation. It is likely that TBS elevates extracellular adenosine levels given evidence that afferent activity releases adenosine in hippocampus (Schubert et al., 1976; Dunwiddie and Masino, 2001) to a degree sufficient to cause homosynaptic and heterosynaptic depression (Mitchell et al., 1993; Brager and Thompson, 2003). These physiological effects appear within $50 \mathrm{~ms}$ and are generated by short afferent bursts (Mitchell et al., 1993), conditions that fall well within the parameters of TBS. Such effects could be larger in the middle-aged slices because of evidence for shifts in adenosine metabolism during aging. Extracellular adenosine levels are reportedly elevated in brains (Murillo-Rodriguez et al., 2004) and hippocampal slices 口SR

(Sperlagh et al., 1997) from aged (22-24 months of age) rats with a trend in the same direction in slices from 12-monthold rats. Possibly related to this, the rate of adenosine removal from slices is slower in aged rats than in young adults (Cunha et al., 2001), and, as shown here, exogenous adenosine has greater effects on synaptic physiology in middle-aged slices. Thus, it is reasonable to propose that age-related changes in adenosine metabolism and clearance combine with release during theta-burst stimulation to generate aberrant extracellular purine concentrations that interact with the size of theta bursts.

Experiments with exogenous adenosine suggest that purine build-up during the theta train probably offsets withintrain burst facilitation rather than actually blocking it. We found that adenosine, presumably acting through presynaptic $\mathrm{A}_{1} \mathrm{Rs}$ to depress the release of glutamate (Dunwiddie and Haas, 1985; Schubert et al., 1986), reduced synaptic responses but did not substantially alter the relationship of a single burst response to the amplitude of the fEPSP used to generate it. More importantly, the facilitation of burst responses during the train was about the same in 750 nM adenosine as it was under control conditions. We suggest that increases in extracellular adenosine, beginning after the first burst of a train, depress the responses that follow the first burst and thereby generate the appearance of blocking burst facilitation.

Three lines of evidence suggest that the age-related effect just described is not responsible for the impaired LTP consolidation found in the str. oriens of middle-aged slices. First, disturbances to theta train facilitation equivalent to those found in the basal dendrites also occurred in the str. radiatum, and these were not accompanied by LTP deficits. If theta-burst responses are suprathreshold for unblocking NMDA receptor-mediated synaptic currents (Larson and Lynch, 1986), presumably they can be reduced somewhat without blocking LTP. This appears to have been the case for the apical dendrites in middle-aged slices, in which a sizeable decrease in burst facilitation was observed in the absence of deficits in LTP induction and consolidation. LTP is more easily induced in the str. oriens than in the str. radiatum (Arai et al., 1994; Leung and Shen, 1995; Roth and Leung, 1995) and thus theta trains that are effective in the latter should be effective in the former. Second, adenosine infusions that had little if any effect on burst facilitation caused a profound depression of LTP consolidation in young adult slices. Although the purine depressed responses, as mentioned, the calculated area of the burst responses during the theta train was reduced by only $\sim 10$ $20 \%$ from control levels. This result suggests that adenosine triggers some event beyond the modulation of theta trains to disrupt LTP consolidation. Third, and in agreement with the point just made, an $\mathrm{A}_{1} \mathrm{R}$ antagonist applied after the theta train completely rescued LTP consolidation in the basal dendrites of middle-aged slices. Pertinent to this, stimulation of $A_{1}$ Rs immediately after LTP induction, by infusing adenosine (Arai et al., 1990b) or through manipulations that increase extracellular adenosine levels (Arai et al., 1990b; Abraham and Huggett, 1997; Huang et al., 
1999), reverses newly formed LTP. This points to the existence of an $\mathrm{A}_{1}$-sensitive disruption process that operates for several minutes after theta stimulation. The results with postinduction DPCPX treatment point to the conclusion that the build-up of adenosine during the theta train persists into the postinduction period and therein initiates the LTP reversal sequence.

Notably missing in the above hypothesis is an explanation for why the postulated age-dependent mechanism works only in the basal dendrites. The basal dendrites were found to be significantly more sensitive than their apical counterparts to the suppressive effects of exogenous adenosine, as were middle-aged relative to young adult slices. These results may indicate that constitutive apical/basal differences interact with generalized age-related changes in adenosine processing to produce conditions in which clearance becomes a problem. Why adenosine in the first instance should have greater effects in the basal dendrites is unknown. Immunochemical analyses indicated that $A_{1} R$ levels are about the same between apical and basal dendritic fields and between middle-aged and young adult animals. There are also reports that rat brain $A_{1} R$ affinity does not change with age (Sperlagh et al., 1997; Cunha et al., 2001). Possibly the enzymes and transport mechanisms that remove extracellular adenosine are less efficient in the str. oriens. There appear to be no reports on adenosine deaminase and adenosine kinase distributions in the hippocampus; thus, evidence relating to this argument is lacking.

Confirmation of the prediction that slices from middle-aged rats exhibit regionally confined changes in adenosine release and/or clearance immediately after theta-burst stimulation would require direct measure of extracellular adenosine concentrations. The measuring technique would have to be (1) sensitive enough to record changes in extracellular adenosine induced by physiological stimulation, (2) able to record locally within discrete dendritic domains, and (3) equipped with sufficient temporal resolution to resolve changes likely occurring during the minutes-long consolidation phase of LTP. Conventional extracellular purine assays, generally requiring collection of slice superfusate, lack the sensitivity, spatial resolution, or temporal resolution to meet such demands (Sperlagh et al., 1997, 2004; Broad et al., 2000). Work now in progress is testing the possibility that recently developed voltametric enzyme biosensors (Dale et al., 2000; Pearson et al., 2001; Llaudet et al., 2003) might be able to satisfy the above criteria.

Finally, the LTP deficits described here suggest that encoding problems are contributors to the memory impairments that emerge between young adulthood and middle age. The memory tasks on which middle-aged rats have deficits (Granger et al., 1996) are dependent on hippocampus and disrupted by agents that block LTP (Morris et al., 1986); thus, it is not unreasonable to assume these memory deficits reflect age-dependent impairments of the type described here. Additional investigation will be required to determine whether other forms of LTP implicated in age-related synaptic plasticity changes, such as NMDAindependent LTP (Shankar et al., 1998), contribute to or interact with the deficits seen here. There are reasons to suspect that the observed middle-aged losses in LTP are a general property of mammalian brain. Recent work suggests that perturbations to adenosine signaling contribute to sleep disturbances in aged animals and humans (Murillo-Rodriguez et al., 2004); if so, adenosine-related impairments to LTP may be as common as age-related disturbances of sleep patterns. In this light, it is encouraging that the middle-aged potentiation deficit did not reflect a lost capacity for LTP expression and was readily offset by disparate drugs. Although results with $A_{1} R$ antagonists suggest that they might ameliorate middle-aged memory deficits, these compounds have undesirable psychomotor stimulant effects (Fisone et al., 2004). Ampakines also restore basal dendritic LTP, do not have such stimulant (or depressive) properties, and have been shown to offset age-related memory losses in rats (Granger et al., 1996) and humans (Lynch et al., 1997). It will be of interest in future studies to determine whether the potency of different ampakine variants to reverse middle-aged LTP deficits predicts their efficacy in treating memory deficits with aging.

\section{References}

Abraham WC, Huggett A (1997) Induction and reversal of long-term potentiation by repeated high-frequency stimulation in rat hippocampal slices. Hippocampus 7:137-145.

Applegate MD, Landfield PW (1988) Synaptic vesicle redistribution during hippocampal frequency potentiation and depression in young and aged rats. J Neurosci 8:1096-1111.

Arai A, Lynch G (1992) Factors regulating the magnitude of long-term potentiation induced by theta pattern stimulation. Brain Res 598:173-184.

Arai A, Larson J, Lynch G (1990a) Anoxia reveals a vulnerable period in the development of long-term potentiation. Brain Res 511:353-357.

Arai A, Kessler M, Lynch G (1990b) The effects of adenosine on the development of long-term potentiation. Neurosci Lett 119:41-44.

Arai A, Black J, Lynch G (1994) Origins of the variations in long-term potentiation between synapses in the basal versus apical dendrites of hippocampal neurons. Hippocampus 4:1-9.

Arai AC, Xia YF, Suzuki E (2004) Modulation of AMPA receptor kinetics differentially influences synaptic plasticity in the hippocampus. Neuroscience 123:1011-1024.

Barnes CA (1979) Memory deficits associated with senescence: a neurophysiological and behavioral study in the rat. J Comp Physiol Psychol 93:74-104.

Barnes CA, McNaughton BL (1985) An age comparison of the rates of acquisition and forgetting of spatial information in relation to long-term enhancement of hippocampal synapses. Behav Neurosci 99:1040-1048.

Barnes CA, Rao G, McNaughton BL (1996) Functional integrity of NMDAdependent LTP induction mechanisms across the lifespan of F-344 rats. Learn Mem 3:124-137.

Barrionuevo G, Schottler S, Lynch G (1980) The effects of repetitive low frequency stimulation on control and "potentiated" synaptic responses in the hippocampus. Life Sci 27:2385-2391.

Brager DH, Thompson SM (2003) Activity-dependent release of adenosine contributes to short-term depression at CA3-CA1 synapses in rat hippocampus. J Neurophysiol 89:22-26.

Broad RM, Fallahi N, Fredholm BB (2000) Nitric oxide interacts with oxygen free radicals to evoke the release of adenosine and adenine nucleotides from rat hippocampal slices. J Auton Nerv Syst 81:82-86.

Capocchi G, Zampolini M, Larson J (1992) Theta burst stimulation is optimal for induction of LTP at both apical and basal dendritic synapses on hippocampal CA1 neurons. Brain Res 591:332-336.

Chang PL, Isaacs KR, Greenough WT (1991) Synapse formation occurs in association with the induction of long-term potentiation in two-year-old rat hippocampus in vitro. Neurobiol Aging 12:517-522.

Costenla AR, de Mendonca A, Ribeiro JA (1999) Adenosine modulates synaptic plasticity in hippocampal slices from aged rats. Brain Res 851:228-234.

Cunha RA, Almeida T, Ribeiro JA (2001) Parallel modification of adenosine extracellular metabolism and modulatory action in the hippocampus of aged rats. J Neurochem 76:372-382.

Dale N, Pearson T, Frenguelli BG (2000) Direct measurement of adenosine release during hypoxia in the CA1 region of the rat hippocampal slice. J Physiol (Lond) 526:143-155.

Davis HP, Small SA, Stern Y, Mayeux R, Feldstein SN, Keller FR (2003) Acquisition, recall, and forgetting of verbal information in long-term memory by young, middle-aged, and elderly individuals. Cortex 39:1063-1091.

Deupree DL, Bradley J, Turner DA (1993) Age-related alterations in potentiation in the CA1 region in F344 rats. Neurobiol Aging 14:249-258.

Diana G, Domenici MR, Loizzo A, Scotti de Carolis A, Sagratella S (1994) Age and strain differences in rat place learning and hippocampal dentate gyrus frequency-potentiation. Neurosci Lett 171:113-116. 
Dunwiddie TV, Haas HL (1985) Adenosine increases synaptic facilitation in the in vitro rat hippocampus: evidence for a presynaptic site of action. J Physiol (Lond) 369:365-377.

Dunwiddie TV, Masino SA (2001) The role and regulation of adenosine in the central nervous system. Annu Rev Neurosci 24:31-55.

Fisone G, Borgkvist A, Usiello A (2004) Caffeine as a psychomotor stimulant: mechanism of action. Cell Mol Life Sci 61:857-872.

Fujii S, Sekino Y, Kuroda Y, Sasaki H, Ito K, Kato H (1997) 8-Cyclopentyltheophylline, an adenosine A1 receptor antagonist, inhibits the reversal of long-term potentiation in hippocampal CA1 neurons. Eur J Pharmacol 331:9-14.

Granger R, Deadwyler S, Davis M, Moskovitz B, Kessler M, Rogers G, Lynch G (1996) Facilitation of glutamate receptors reverses an age-associated memory impairment in rats. Synapse 22:332-337.

Herndon JG, Moss MB, Rosene DL, Killiany RJ (1997) Patterns of cognitive decline in aged rhesus monkeys. Behav Brain Res 87:25-34.

Huang CC, Liang YC, Hsu KS (1999) A role for extracellular adenosine in time-dependent reversal of long-term potentiation by low-frequency stimulation at hippocampal CA1 synapses. J Neurosci 19:9728-9738.

Kaibara T, Leung LS (1993) Basal versus apical dendritic long-term potentiation of commissural afferents to hippocampal CA1: a current-source density study. J Comp Neurol 13:2391-2404.

Kramár EA, Lynch G (2003) Developmental and regional differences in the consolidation of long-term potentiation. Neuroscience 118:387-398.

Kramár EA, Bernard JA, Gall CM, Lynch G (2003) Integrins modulate fast excitatory transmission at hippocampal synapses. J Biol Chem 278:10722-10730.

Kramár EA, Lin B, Lin CY, Arai AC, Gall CM, Lynch G (2004) A novel mechanism for the facilitation of theta-induced long-term potentiation by brain-derived neurotrophic factor. J Neurosci 24:5151-5161.

Lanahan A, Lyford G, Stevenson GS, Worley PF, Barnes CA (1997) Selective alteration of long-term potentiation-induced transcriptional response in hippocampus of aged, memory-impaired rats. J Neurosci 17:2876-2885.

Landfield PW, Lynch G (1977) Impaired monosynaptic potentiation in in vitro hippocampal slices from aged, memory-deficient rats. J Gerontol 32:523-533.

Landfield PW, McGaugh JL, Lynch G (1978) Impaired synaptic potentiation processes in the hippocampus of aged, memory-deficient rats. Brain Res 150:85-101.

Larson J, Lynch G (1986) Induction of synaptic potentiation in hippocampus by patterned stimulation involves two events. Science 232:985-988.

Larson J, Wong D, Lynch G (1986) Patterned stimulation at the theta frequency is optimal for the induction of hippocampal long-term potentiation. Brain Res 368:347-350.

Larson J, Xiao P, Lynch G (1993) Reversal of LTP by theta frequency stimulation. Brain Res 600:97-102.

Lee KS, Schubert P, Reddington M, Kreutzberg GW (1986) The distribution of adenosine A1 receptors and 5'-nucleotidase in the hippocampal formation of several mammalian species. J Comp Neurol 246:427-434.

Leung LS, Shen B (1995) Long-term potentiation at the apical and basal dendritic synapses of CA1 after local stimulation in behaving rats. J Neurophysiol 73:1938-1946.

Leung LS, Shen B (1999) N-methyl-D-aspartate receptor antagonists are less effective in blocking long-term potentiation at apical than basal dendrites in hippocampal CA1 of awake rats. Hippocampus 9:617-630.

Llaudet E, Botting NP, Crayston JA, Dale N (2003) A three-enzyme microelectrode sensor for detecting purine release from central nervous system. Biosens Bioelectron 18:43-52.

Lynch G (2002) Memory enhancement: the search for mechanism-based drugs. Nat Neurosci 5:1035-1038.

Lynch G, Granger R, Ambros-Ingerson J, Davis CM, Kessler M, Schehr R (1997) Evidence that a positive modulator of AMPA-type glutamate receptors improves delayed recall in aged humans. Exp Neurol 145:89-92.

Meneses A, Manuel-Apolinar L, Rocha L, Castillo E, Castillo C (2004) Expression of the 5-HT receptors in rat brain during memory consolidation. Behav Brain Res 152:425-436.

Mitchell JB, Lupica CR, Dunwiddie TV (1993) Activity-dependent release of endogenous adenosine modulates synaptic responses in the rat hippocampus. J Neurosci 13:3439-3447.

Moore CI, Browning MD, Rose GM (1993) Hippocampal plasticity induced by primed burst, but not long-term potentiation, stimulation is impaired in area CA1 of aged Fischer 344 rats. Hippocampus 3:57-66.
Morris RG, Anderson E, Lynch GS, Baudry M (1986) Selective impairment of learning and blockade of long-term potentiation by an N-methyl-Daspartate receptor antagonist, AP5. Nature 319:774-776.

Murillo-Rodriguez E, Blanco-Centurion C, Gerashchenko D, Salin-Pascual RJ, Shiromani PJ (2004) The diurnal rhythm of adenosine levels in the basal forebrain of young and old rats. Neuroscience 123:361-370.

Nicoll RA (2003) Expression mechanisms underlying long-term potentiation: a postsynaptic view. Philos Trans R Soc Lond B Biol Sci 358:721-726.

Norris CM, Korol DL, Foster TC (1996) Increased susceptibility to induction of long-term depression and long-term potentiation reversal during aging. J Neurosci 16:5382-5392.

Oler JA, Markus EJ (1998) Age-related deficits on the radial maze and in fear conditioning: hippocampal processing and consolidation. Hippocampus 8:402-415.

Omrani A, Fathollahi Y (2003) Reversal of pentylenetetrazol-induced potentiation phenomenon by theta pulse stimulation in the CA1 region of rat hippocampal slices. Synapse 50:83-94.

Park DC, Welsh RC, Marshuetz C, Gutchess AH, Mikels J, Polk TA, Noll DC, Taylor SF (2003) Working memory for complex scenes: age differences in frontal and hippocampal activations. J Cogn Neurosci 15:1122-1134.

Pearson T, Nuritova F, Caldwell D, Dale N, Frenguelli BG (2001) A depletable pool of adenosine in area CA1 of the rat hippocampus. J Neurosci 21:2298-2307.

Rivkees SA, Price SL, Zhou FC (1995) Immunohistochemical detection of A1 adenosine receptors in rat brain with emphasis on localization in the hippocampal formation, cerebral cortex, cerebellum, and basal ganglia. Brain Res 677:193-203.

Roth LR, Leung LS (1995) Difference in LTP at basal and apical dendrites of CA1 pyramidal neurons in urethane-anesthetized rats. Brain Res 694:40-48.

Sah P, Bekkers JM (1996) Apical dendritic location of slow afterhyperpolarization current in hippocampal pyramidal neurons: implications for the integration of long-term potentiation. J Neurosci 16:4537-4542.

Sans N, Petralia RS, Wang YX, Blahos II J, Hell JW, Wenthold RJ (2000) A developmental change in NMDA receptor-associated proteins at hippocampal synapses. J Neurosci 20:1260-1271.

Schubert P, Lee K, West M, Deadwyler S, Lynch G (1976) Stimulationdependent release of ${ }^{3} \mathrm{H}$-adenosine derivatives from central axon terminals to target neurones. Nature 260:541-542.

Schubert P, Heinemann U, Kolb R (1986) Differential effect of adenosine on pre- and postsynaptic calcium fluxes. Brain Res 376:382-386.

Shankar S, Teyler TJ, Robbins N (1998) Aging differentially alters forms of long-term potentiation in rat hippocampal area CA1. J Neurophysiol 79:334-341.

Shearman LP, Weaver DR (1997) [ $\left.{ }^{125} \mathrm{I}\right] 4$-aminobenzyl-5'-N-methylcarboxamidoadenosine ( $\left.\left.{ }^{125} \mathrm{I}\right) \mathrm{AB}-\mathrm{MECA}\right)$ labels multiple adenosine receptor subtypes in rat brain. Brain Res 745:10-20.

Sloane JA, Pietropaolo MF, Rosene DL, Moss MB, Peters A, Kemper T, Abraham CR (1997) Lack of correlation between plaque burden and cognition in the aged monkey. Acta Neuropathol (Berl) 94:471-478.

Sperlagh B, Zsilla G, Baranyi M, Kekes-Szabo A, Vizi ES (1997) Agedependent changes of presynaptic neuromodulation via A1-adenosine receptors in rat hippocampal slices. Int J Dev Neurosci 15:739-747.

Sperlagh B, Baranyi M, Hasko G, Vizi ES (2004) Potent effect of interleukin-1 beta to evoke ATP and adenosine release from rat hippocampal slices. J Neuroimmunol 151:33-39.

Staubli U, Chun D (1996) Proactive and retrograde effects on LTP produced by theta pulse stimulation: mechanisms and characteristics of LTP reversal in vitro. Learn Mem 3:96-105.

Swanson TH, Drazba JA, Rivkees SA (1995) Adenosine Al receptors are located predominantly on axons in the rat hippocampal formation. J Comp Neurol 363:517-531.

Tombaugh GC, Rowe WB, Chow AR, Michael TH, Rose GM (2002) Thetafrequency synaptic potentiation in CA1 in vitro distinguishes cognitively impaired from unimpaired aged Fischer 344 rats. J Neurosci 22:9932-9940.

Ward MT, Oler JA, Markus EJ (1999) Hippocampal dysfunction during aging I: deficits in memory consolidation. Neurobiol Aging 20:363-372.

Watabe AM, O'Dell TJ (2003) Age-related changes in theta frequency stimulation-induced long-term potentiation. Neurobiol Aging 24:267272 . 